\title{
Parametric Design of Straight Bevel Gears Based on Solidworks
}

\author{
Shan Yuxia \\ College of Mechanical and Electronic Engineering, \\ Nanjing Forestry University \\ Nanjing,China ,210037 \\ yuxiashan@126.com
}

\author{
Zhang Wei \\ College of Mechanical and Electronic Engineering, \\ Nanjing Forestry University \\ Nanjing,China ,210037
}

\begin{abstract}
The Visual Basic program of standard straight bevel gear's parametric design based on Solidworks software was completed. The geometric features of bevel gears were analyzed, parametric design variables were defined, each control point was determined in Cartesian Coordinate System. In development of the system, the API functions such as CreatLine, FeatureRevolve,FeatureCut, CreatePlaneAtAngle3, InsertCutBlend and FeatureCircularPattern were used; the involute profiles of bevel gear at large end were fit by splines. The parametric design of bevel gears may provide a basis for further finite element stress analysis or assembly.
\end{abstract}

Keywords- Bevelgears; Solidworks, API; Parametric Design

\section{INTRODUCTION}

A bevel gear is one of the most fundamental types of gear, it is widely used in power transmission systems of aircrafts, automobiles and engineering machines, etc..Bevel gears are cut on conical blanks to be used to transmit motion between intersecting shafts. Straight bevel gears are the most economical of the various bevel gears, owing to their ease of manufacture.

Accurate 3D modeling of gears are critically important to their FEM analysis, motion or dynamic simulation and CNC production. Developing program module for parametric modeling of gear in 3D design software would make the design more efficient and quality.

As a 3D mechanical design software, SolidWorks has been extensively used due to its Windows-native design environment, powerful assembly capabilities, ease-of-use, and affordable price[1]. Moreover, Solidworks can be further developed conveniently by its API(Application Programming Interface) and $\mathrm{VBA}($ Visual Basic for Applications) or $\mathrm{VC}++$, Visual Basic, etc..

The API is an OLE programming interface to SolidWorks, it contains hundreds of functions that can be called from VBA, VB.NET, Visual $\mathrm{C}++6.0$, and Visual $\mathrm{C}++$, etc.. These functions provide direct access to SolidWorks functionality such as creating a line, cutting a hole, or verifying the parameters of a surface[2].

Many researches on cylindrical gear's parametric design have been done based on Solidworks[3-5], but there is few research on bevel gear's parametric design, because of its special geometric structure. Although the study have been done by LI Jun-wei and PAN Yu-tian, but some mistakes were found from its running results, such as the value of the pitch cone angle and the teeth profiles[6]. In this paper, a parametric design system for straight bevel gears was developed based on Solidworks 2008 by use of Visual Basic language and the API, which provides designers with an interactive computer-aided design environment, it will make the straight bevel gear's 3D design easier, faster and more accurate.

\section{GeOMEtry Of Straight Bevel GeArs}

The geometry of bevel gears is shown in Fig.1.They have teeth that are straight and tapered, if extended inward, the teeth would intersect at a common point $\mathrm{O}[7]$. The shaft angle is $90^{\circ}$, which is the sum of the pitch cone angle of gear $2 \delta_{2}$ and pinion $1 \delta_{1}$. In order to secure uniform bearing along the tooth, the face width $b$ is generally not made longer than one-third of the pitch cone length $R$, usually $b=\varphi_{\mathrm{R}} \cdot R$, $\varphi_{\mathrm{R}}=0.25 \sim 0.3$, the gear ratio is given as follows:

$$
i=\frac{z_{2}}{z_{1}}=\tan \delta_{2}=\cot \delta_{1}
$$

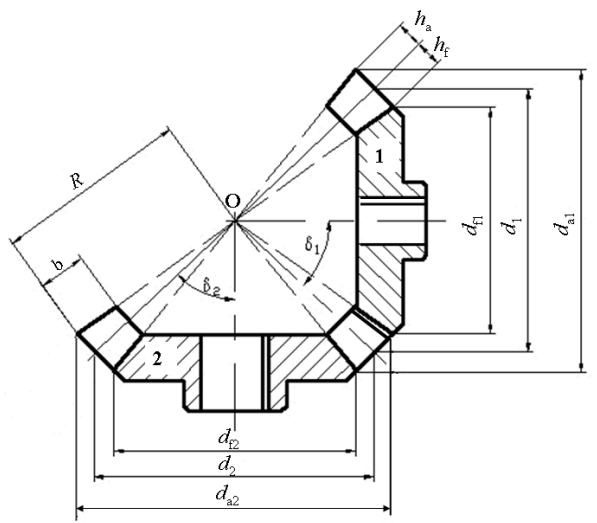

Figure 1. The geometry of bevel gears

As shown in Fig.1 and Fig.2, the size and shape of the straight bevel gear's teeth are defined at the large end, on the back cone, which have standard involute profiles. The length of back cone $r_{\mathrm{e}}$ has relationship with the pitch diameter $d$ as equation (2); it is equal to the pitch circle radius of bevel gear's virtual spur gear.

$$
r_{e}=\frac{d}{2 \cos \delta}
$$




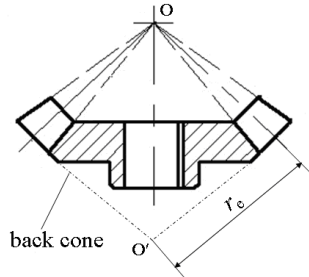

(a) Back cone

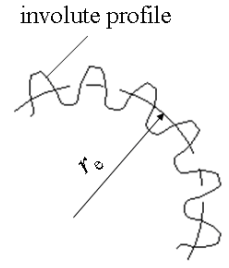

(b) Involute profiles of teeth
Figure 2. Back cone and shape of teeth on it

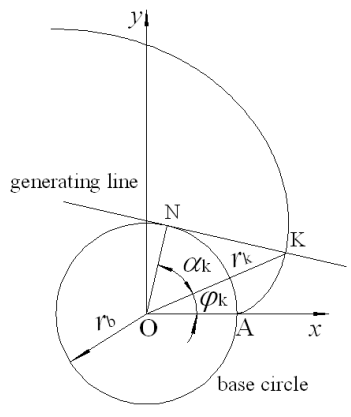

Figure 3. Generation of an involute curve

An involute curve is the locus of a point on the generating line, as the line rolls without slipping along a base circle[8], see Fig.3. Gear teeth are cut in the shape of an involute curve between the base and the addendum circles, while the part of the tooth between the base and dedendum circles is generally a radial line[9]. In Cartesian Coordinate System, the involute curve is expressed as follows:

$$
\left\{\begin{array}{l}
x=r_{b}(\cos \theta+\theta \sin \theta) \\
y=r_{b}(\sin \theta-\theta \cos \theta)
\end{array}\right.
$$

Where $r_{\mathrm{b}}$ is radius of the base circle, $\theta$ is the spread angle $\theta=\operatorname{tg} \alpha_{\mathrm{k}}$. Referring to Fig.3, for gear tooth profile the definition interval of $\theta$ is $0 \leq \theta \leq \sqrt{\left(d_{a} / d_{b}\right)^{2}-1}$. Note especially that when determine the involute tooth profile of bevel gear at large end, the geometric parameters of its virtual spur gear at large end should be substituted.

\section{AchiEvement Of Straight BeVEl GeAR'S PARAMETRIC DESIGN}

\section{A. Determination of design variables}

According to geometric features of straight bevel gears stated above, and design equations for bevel gears which are detailed in Mechanical Design Manual[10], the module $m$ at large end, tooth number $z_{1}$ of pinion, face width factor $\varphi_{\mathrm{R}}$ and gear ratio $i$ are determined as parametric design variables. The pressure angle $\alpha$, addendum height factor $h_{\mathrm{a}}{ }^{*}$ and tip clearance factor $c^{*}$ is set as the standard value of $20^{\circ}, 1$ and 0.2 , respectively. The interactive interface was designed as Fig.4, where the standard values of module $m$ in accord with GB12368-90 can be selected directly by the popup-list tile.

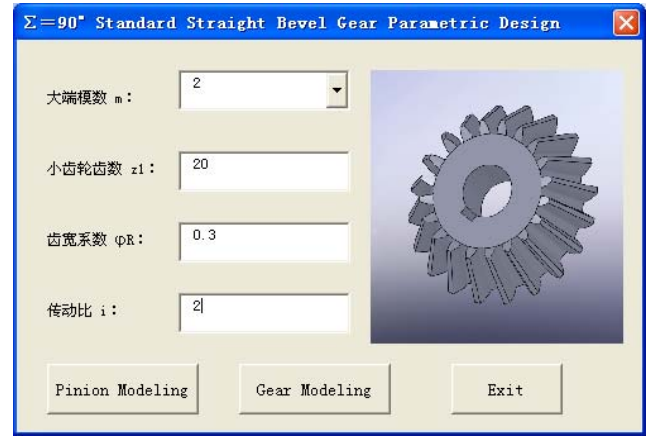

Figure 4. Parameters entering interface

\section{B. Coordinates determination of control points}

Before implementing parametric design by Visual Basic codes and API functions, some coordinates of control points must be determined, according to the design variables stated above and the topological properties of bevel gears. Thus, a Cartesian Coordinate System was set up, as shown in Fig.5, whose origin is at the apex of the back cone. In this coordinate system all the control points were determined, referring to Fig. 5 and Tab.1.

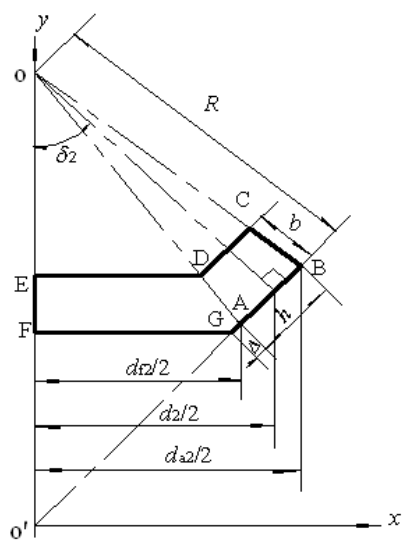

Figure 5. Coordinate frame for cross section of a bevel gear

TABLE I. COORDINATES DETERMINATION OF CONTROL POINTS

\begin{tabular}{|c|c|c|}
\hline points & $\boldsymbol{x}$ & $\boldsymbol{y}$ \\
\hline $\mathrm{O}^{\prime}$ & 0 & 0 \\
\hline $\mathrm{O}$ & $x_{O}=0$ & $y_{O}=R / \cos \delta_{2}$ \\
\hline $\mathrm{A}$ & $x_{a}=d_{f 2} / 2$ & $y_{a}=d_{f 2} * i / 2$ \\
\hline $\mathrm{B}$ & $x_{b}=d_{a 2} / 2$ & $y_{b}=d_{a 2} * i / 2$ \\
\hline $\mathrm{C}$ & $x_{c}=\left(1-\varphi_{R}\right) * h * \cos \delta_{2}+x_{d}$ & $y_{c}=\left(1-\varphi_{R}\right) * h^{*} \sin \delta_{2}+y_{d}$ \\
\hline $\mathrm{D}$ & $x_{d}=\left(1-\varphi_{R}\right) * d_{f} / 2$ & $y_{d}=y_{e}$ \\
\hline $\mathrm{E}$ & $x_{e}=0$ & $y_{e}=\left(y_{O}-y_{a}\right) * \varphi_{R}+y_{a}$ \\
\hline $\mathrm{F}$ & $x_{f}=0$ & $y_{f}=y_{g}$ \\
\hline $\mathrm{G}$ & $x_{g}=\left(1-\frac{\Delta}{d_{2} / 2 / \cos \delta_{2}-h_{f}}\right) * x_{a}$ & $y_{g}=\left(1-\frac{\Delta}{d_{2} / 2 / \cos \delta_{2}-h_{f}}\right) * y_{a}$ \\
\hline
\end{tabular}




\section{Procedures of bevel gear's parametric design}

According to the study results above, parametric design system for straight bevel gears were achieved by use of API functions based on Solidworks 2008. The flow chart of this work is shown as Fig.6.

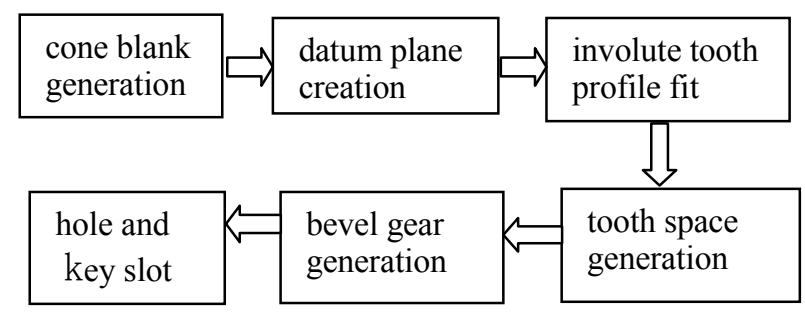

Figure 6. Flow chart of parametric design

Firstly, a two-dimensional cross section graph of cone blank(see Fig.5) was sketched, CreatLine function was applied according to coordinates of control points in tab.1;

Secondly, the sketch was revolved by FeatureRevolve function to generate a cone blank; and a datum plane was created by CreatePlaneAtAngle 3 function, which is tangential to the back cone, as shown in Fig.8(a).

Thirdly, two involute curves for toothspace profile were fitted by splines on the datum plane, enclosed by arcs, see Fig.7.

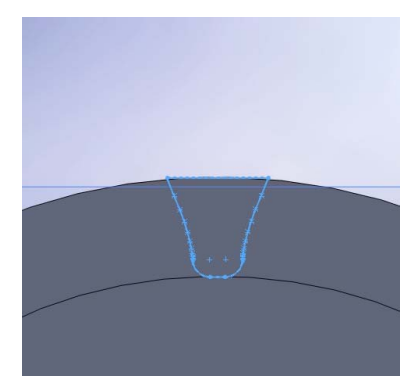

Figure 7. Involute toothspace profile

Further, a point was created at apex $\mathrm{O}$ of the pitch cone by CreatPoint function, together with the toothspace curves, a toothspace was generated by function of InsertCutBlend, see Fig.8(a).

Lastly, the toothspace was arrayed by FeatureCircularPattern2 function, so far the modeling is almost finished; basing on it, some holes and key slot can be easily cut by FeatureCut function, see Fig.8(b).

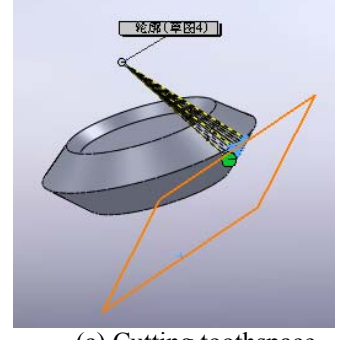

(a) Cutting toothspace

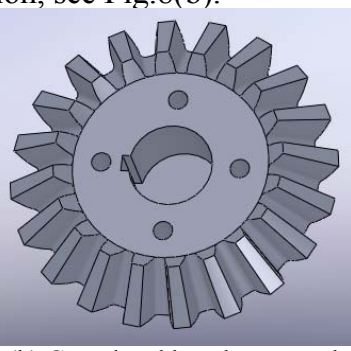

(b) Completed bevel gear mode
Figure 8. Modeling of straight bevel gear

\section{Usage and results}

Run the macros programmed by Visual Basic codes and API functions in Solidworks part environment, a standard straight bevel gear model would be automatically and quickly generated after interactive operation. A pair of bevel gears created by running the macros was assembled, it showed that they fit well, see Fig.9.

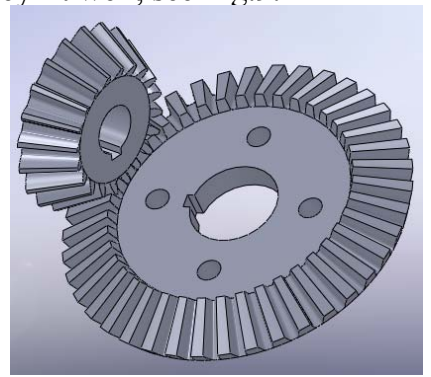

Figure 9. Assembled bevel gears

\section{SUMMARIES}

In this study, structural features of straight bevel gear were analyzed; design variables were specified, and then the interactive interface was designed; Further, a Cartesian Coordinate System was set up and coordinates of control points were determined according to its geometric relationship. Through Visual Basic language and Solidworks API functions, parametric design of straight bevel gear has been implemented, running results showed that the parametric modeling of bevel gear was speedy and accurate, which will facilitate the 3D modeling of straight bevel gears in Solidworks.

However, the methodology proposed in this paper is mainly addressed for straight bevel gears which intersect at right angle, but it provides references for straight bevel gears at other angles and spiral bevel gears. Based on it, the next work is to study the parametric design of them in Solidworks.

\section{REFERENCES}

[1] L. Kong; J.Y.H. Fuh; K.S. Lee, etc.. A Windows-native 3D Plastic Injection Mold Design System. Journal of Materials Processing Technology[J], 2003.139, PP81-89.

[2] Solidworks Corporation. Solidworks Advanced Training:Further Development \& API[M]. Beijing:China Machine Press,2009,PP1-121.

[3] ZHANG Yan-fu; XIA Chun. A Method of Involute Gear 3d Mode1 With SolidWorks. Machinery Design \& Manufacture[J], 2005.11, PP61-62.

[4] XUE Chen.Study of gear 3D Design Method Based on Solidworks.Development and Innovation of Machinery \& Electrical Products[J], 2010.5, PP88-90.

[5] XU Jie.Research on Gear's 3D Parametrical Design Based on Solidworks.Machinery \& Electricity Messages[J] ,2010.30, PP130131.

[6] LI Jun-wei, PAN Yu-tian. Parametric Design and Finite Element Analysis of Straight Bevel Gear Based on SolidWorks[J].Modern Production Engineering,2009.12,PP52-55.

[7] M.F.Spotts; T.E.Shoup. Design of Machine Elements[M]. Beijing:China Machine Press,2003,PP596-600.

[8] Mathematic Manual Compiling Group. Mathematic Manual[M]. Beijing:Higher Education Press, 1979,PP380-381. 
The 2nd International Conference on Computer Application and System Modeling (2012)

[9] Ansel C.Ugural.Mechanical Design(An Integrated Approach)[M]. Chongqing:Chongqing University Press,2005.1,PP110-111.
[10] WU Zong-ze.Mechanical Elements Design Manual[M].Beijing: China Machine Press,2004,PP703-707.

Published by Atlantis Press, Paris, France.

(C) the authors 